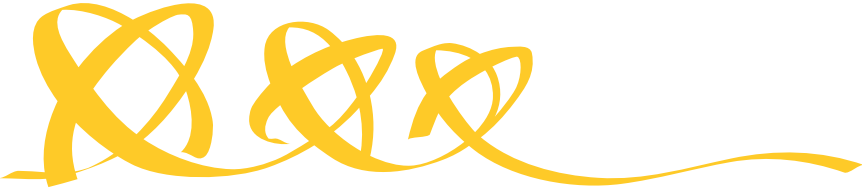 COMMUNICATIONS PHYSICS
}

COMMENT

https://doi.org/10.1038/s42005-019-0130-z

\section{The dawn of non-Hermitian optics}

\author{
Ramy El-Ganainy ${ }^{1,2}$, Mercedeh Khajavikhan ${ }^{3}$, Demetrios N. Christodoulides $^{3}$ \& \\ Sahin K. Ozdemir ${ }^{4,5}$
}

Recent years have seen a tremendous progress in the theory and experimental implementations of non-Hermitian photonics, including all-lossy optical systems as well as parity-time symmetric systems consisting of both optical loss and gain. This progress has led to a host of new intriguing results in the physics of light-matter interactions with promising potential applications in optical sciences and engineering. In this comment, we present a brief perspective on the developments in this field and discuss possible future research directions that can benefit from the notion of non-Hermitian engineering.

\section{Introduction}

The notion of non-Hermitian parity-time (PT) reversal symmetric Hamiltonians having real spectra was first conceived within the context of quantum physics ${ }^{1,2}$. The introduction of this concept later in optics ${ }^{3,4}$, has led to an explosion of research activities aimed to explore some of the exotic features displayed by such non-conservative systems. In general, such configurations can be synthesized by establishing an even distribution in the real part of the refractive index while imposing an antisymmetric gain/loss profile, associated with the imaginary part of the refractive index.

For example, despite being non-Hermitian, PT symmetric photonic structures can exhibit either entirely real spectra or complex conjugate eigenvalue pairs. In the first regime, the eigenstates also respect PT symmetry (i.e., the system is in an exact PT phase) while in the latter, PT symmetry is broken with the optical intensities associated with the eigenstates being more concentrated either in the gain or in the loss region ${ }^{4}$. The ability to design and implement photonic systems operating in these domains has led to new light-wave dynamics and unusual optical effects such as unidirectional invisibility ${ }^{5,6}$, laser self-termination ${ }^{7,8}$ and complex Bloch

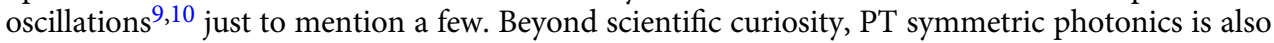
paving the way towards new technological innovations. For example, PT symmetry can be used to engineer the spectral or the spatial properties of laser emission ${ }^{11-15}$.

\footnotetext{
${ }^{1}$ Department of Physics and Henes Center for Quantum Phenomena, Michigan Technological University, Houghton, MI 49931, USA. ${ }^{2}$ Department of Electrical and Computer Engineering, Michigan Technological University, Houghton, MI 49931, USA. ${ }^{3}$ College of Optics \& Photonics-CREOL, University of Central Florida, Orlando, FL 32816, USA. ${ }^{4}$ Department of Engineering Science and Mechanics \& Materials Research Institute, The Pennsylvania State University, University Park, PA 16802-6812, USA. ${ }^{5}$ Materials Research Institute, The Pennsylvania State University, University Park, PA 16802-6812, USA. Correspondence and requests for materials should be addressed to R.E-G. (email: ganainy@mtu.edu)
} 
The intense research activity around PT symmetry in optics ${ }^{16-20}$ has in turn led to the more general framework of nonHermitian photonics that seeks to use a judicious engineering of the interplay between gain, loss and optical index distributions in order to attain new functionalities. While the notion of PTsymmetry requires the presence of gain in the system to compensate for losses, non-Hermitian photonics does not necessarily rely on the presence of gain. Non-Hermiticity covers all open physical systems, regardless of the presence or the absence of amplification, including loss-only arrangements.

\section{Non-Hermitian optics toolbox}

These new overarching concepts are currently expanding the toolbox of photonics by exploiting the complex refractive index landscape, thus enriching the design parameter space (see Fig. 1). Importantly, numerous platforms, ranging from waveguide/cavity arrangements ${ }^{4,11,21}$ and fiber optics ${ }^{22}$ to metamaterial ${ }^{6}$, and microwave systems ${ }^{23}$, are readily available for building nonHermitian photonics. This opens the door for a wide range of applications and future technologies such as optical sensors 24,25 , microlasers ${ }^{11,12}$, nonreciprocal light propagation ${ }^{26}$, telemetry ${ }^{27}$, and microscopic thermal mapping ${ }^{28}$.

When a non-Hermitian optical system is steered in the complex eigenenergy landscape by varying one or more of its parameters, it can be brought to the vicinity of a special type of spectral singularity called an exceptional point (EP). Figure 2 illustrates some of the features of EPs using an archetypal discrete Hamiltonian. Exceptional points differ from Hermitian degeneracies known as diabolic points (DPs): at a DP, the eigenvalues are degenerate but the corresponding eigenstates are orthogonal whereas at an EP not only the eigenvalues but also their corresponding eigenstates become identical, indicating a collapse of the eigenspace dimensionality at an EP. This in turn renders the system very sensitive to any external perturbation. As it turns out, this responsivity becomes even more pronounced as the order of the exceptional point (defined by the number of coalescing eigenvalues and eigenstates at the EP) is increased. This can be better understood by inspecting the Riemann surface associated with the eigenvalues of nonHermitian Hamiltonians, where EPs appear as edge points on the lines connecting two sheets. For example, Fig. 2a, b depicts such surface for an archetypical PT symmetric dimer where the

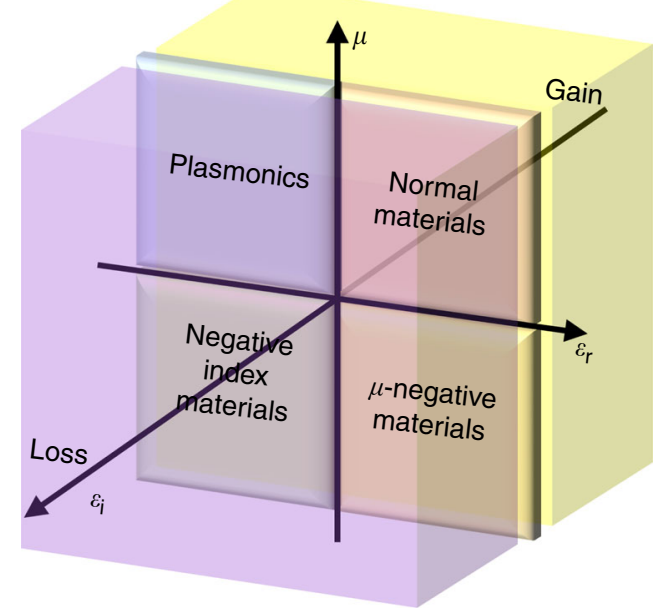

Fig. 1 No-Hermitian photonics. Non-Hermiticity expands the toolbox for integrated photonics by enlarging the parameter space into the complex domain (yellow and violet regions along the positive/negative $\varepsilon_{i}$ axis representing loss or gain) locations of EPs are also indicated ${ }^{17}$. Along the projection marked by white lines (where the Hamiltonian is PT symmetric), the eigenvalues bifurcate as the system crosses the EP. Importantly, for small deviations from the EP, this eigenvalue splitting is stronger than the splitting in Hermitian systems. Figure $2 \mathrm{c}$ shows the behavior of the two eigenvectors highlighting the non-Hermitian degeneracy of their coalescence by using the concept of Hermitian angle ${ }^{29}$. These features, together with a recent proposal for utilizing bosonic algebra to engineer discrete photonic arrays having higher order EPs, was recently employed to demonstrate optical and radio frequency (RF) sensors with superior performance $24,25,27$ compared to those operating based on diabolic points. Interestingly, EPs can affect the dynamics of the system even when they are away from the operating point as has been demonstrated recently by the work on dynamic encircling of these singularities ${ }^{30-32}$.

\section{Future perspective}

A particular field that can benefit significantly from nonHermiticity is nonlinear optics. Recently, non-Herrmitian engineering was proposed as a means for building on-chip light sources $^{33}$ that rely on coherent wave-mixing processes without the need for restrictive phase-matching conditions (momentum conservation) - which represents a necessary requirement for efficient light generation in standard nonlinear optical systems. Within the context of four-wave mixing where two pump photons fuse together to produce one signal and one idler photon, this proposition relies on the spectral and spatial engineering of optical losses (absorption or radiation) in order to sweep the idler component out of the system, thus leaving only the signal wave to enjoy amplification in an irreversible fashion ${ }^{34}$. This concept is schematically illustrated in Fig. 3. This opens up new possibilities for nonlinear processes, because nonlinear effects in Hermitian settings are by nature reversible, i.e., when the idler and signal reach a high enough intensity, they can recombine to create pump photons which then sets a limit on the attainable signal or idler powers. Sweeping the idler component out of the system prevents this recombination (backward process) and forces the nonlinear wave-mixing to proceed in the forward direction such that signal power increases as the pump is increased. Different implementations for this concept include hybrid dielectric plasmonic waveguides $^{35}$, coupled cavity systems utilizing the Vernier effect $^{36}$, and Bragg grating loaded waveguides ${ }^{37}$. A particularly challenging task in all these systems is to achieve the desired spectral/spatial loss engineering without sacrificing the modal overlap necessary for efficient nonlinear interactions. While similar effects related to loss-induced modulation instabilities have been investigated in fiber optics setups ${ }^{38}$, this approach becomes particularly interesting when considering semiconductor platforms with the potential of building miniaturized nonlinear light sources.

Currently, the impact of non-Hermitian engineering is rapidly expanding into different research directions. On the one hand, nonHermiticity combined with other quantum-inspired symmetries and technologies can be used to observe more exotic effects. Exemplifying this direction is the recent work on supersymmetric laser arrays ${ }^{39,40}$ and the experimental observation of topological lasers in both one and two dimensional systems ${ }^{41-45}$. In the former, pseudo global phase matching, which is engineered by using supersymmetry transformations, is used in conjunction with loss engineering to spoil the quality factor of all the undesired supermodes of a laser array in order to force the system to lase in the inphase fundamental supermode. In the latter, the judicious introduction of loss and gain in a 1D Su-Schrieffer-Heeger (SSH) lattice replaces the usual chiral symmetry of the topological array with 

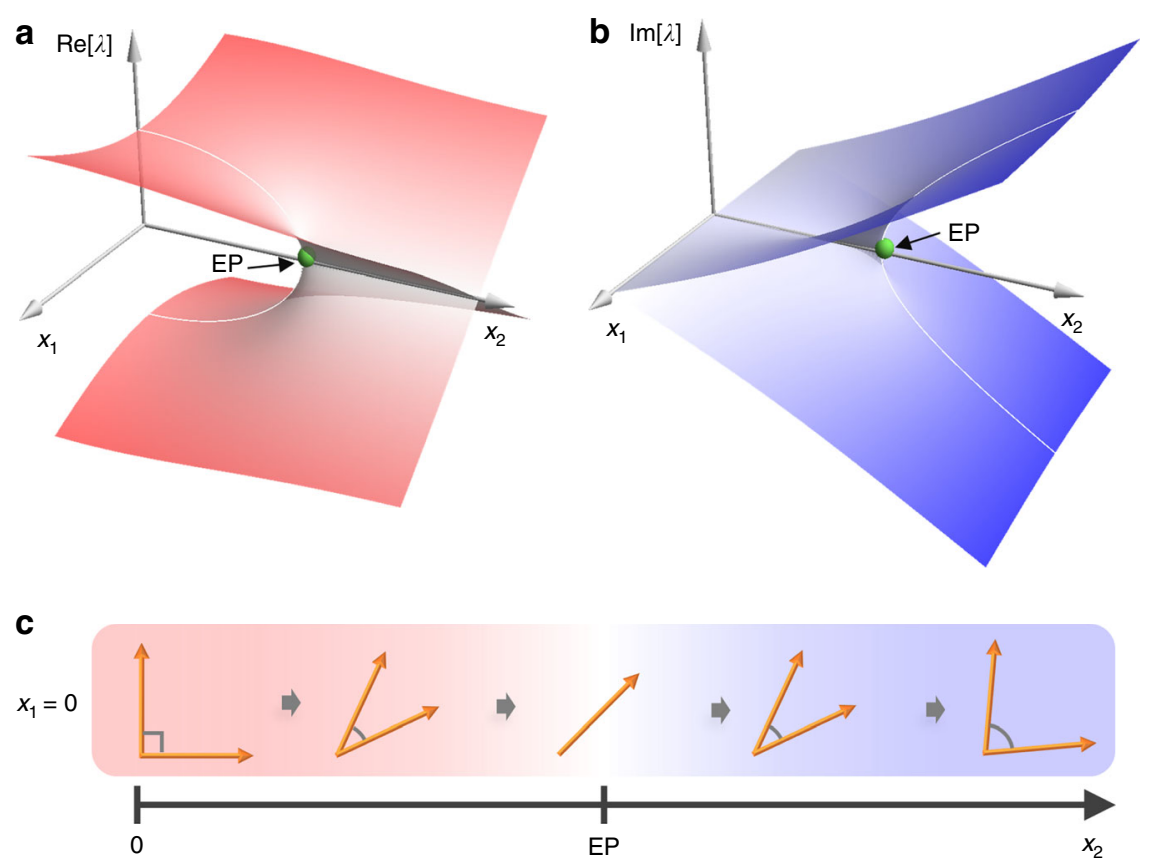

Fig. 2 Exceptional points. $\mathbf{a}$ and $\mathbf{b}$ plot the real and imaginary parts of the eigenvalues (Riemann surface) associated with an archetypal $2 \times 2$ non-Hermitian Hamiltonian $H=\left(\begin{array}{cc}x_{1}+i x_{2} & 1 \\ 1 & -x_{1}-i x_{2}\end{array}\right)$ (representing coupled waveguides or resonators for instance) having an exceptional point (EP) of order two, as a function of two parameters $x_{1,2}$. The singular point between the two sheets of the Riemann surface is called an EP. c Presents the Hermitian angle 29 between the two eigenvectors of $H$ as a function of the non-Hermitian parameter $x_{2}$ when $x_{1}=0$. At the EP, not only the eigenvalues but also the eigenvectors coalesce and become identical. Additionally, the Taylor series expansion fails at that point. These properties equip non-Hermitian systems with very exotic behavior such as loss-induced gain, mode selectivity, loss-enabled phase matching, to mention just a few examples (see ref. ${ }^{17,18}$ for more details)

\section{a}

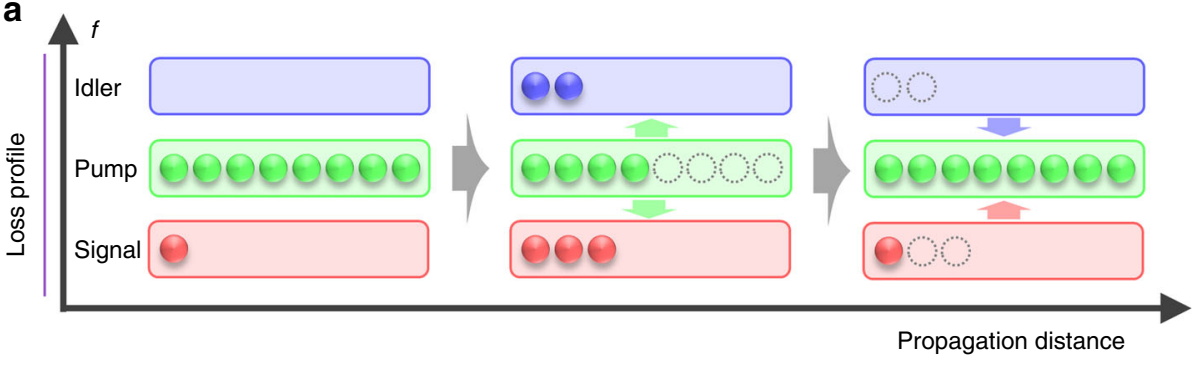

b

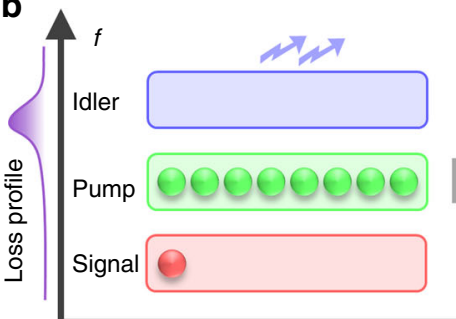

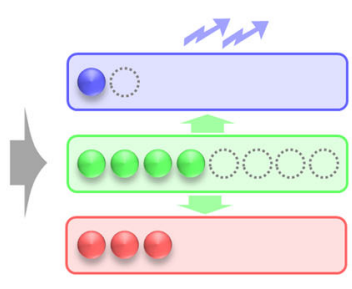

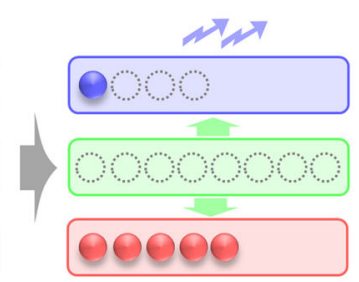

Propagation distance

Fig. 3 Non-Hermitian parametric amplification. Schematic illustration of the process of four wave-mixing when the phase matching condition is violated in two different cases: $\mathbf{a}$ when the losses of all the components are neglected; and $\mathbf{b}$ when the idler loss is appreciable while the pump and signal loss are negligible. In the first scenario, the process is reversible as a function of the propagation distance: two pump photons can be combined to produce a signal and an idler photons, which upon propagation acquire different phases that allows them to combine and reproduce the pump photons again. In the latter case (b), the idler loss sweeps the idler photons out of the system, leaving only the signal photons. As a result, the process becomes irreversible. This leads to the amplification of the signal beam even in the absence of phase matching. The conversion efficiency between the different components depend on the exact frequencies involved and the details of the loss profile 33,34 
charge-conjugation symmetry, which in turn allows the system to lase in a pre-selected topologically protected defect state even when the whole structure is uniformly pumped. On the other hand, the notion of non-Hermitian engineering is applied in numerous physical platforms other than optics such as electronic ${ }^{46}$ and microwave ${ }^{23}$ with potential applications in wireless power transfer ${ }^{47}$ and telemetry sensing ${ }^{27}$. Along this line, two directions promise major advances in light sciences and technologies: optomechanics and plasmonics. Concepts from non-Hermitian engineering have already been used in theoretical explorations in these fields, showing how PT symmetric systems can be integrated with plasmonics structures ${ }^{48}$, and predicting emergence of high-order EPs in optomechanical systems, enhanced optomechanical interactions and cooling rates ${ }^{49}$, as well as thresholdless phonon lasing ${ }^{50}$. The experimental demonstration of such predictions can lead to technological advances. For example, a recent experimental work has demonstrated the broadening of the linewidth of a phonon laser operating in the vicinity of an exceptional point (analogous to Petermann factor in photon lasers physics) and the possibility of tuning the linewidth of phonon lasers by operating them close to or far from exceptional points ${ }^{51}$.

The research area of non-Hermitian photonics is relatively new. Compared to other more mature fields such as photonic crystals or metamaterials, it is still largely unexplored. Novel theoretical ideas combined with new experimental schemes are expected to produce more surprising results, leading to altogether different, previously unknown, means for controlling light-matter interactions both in the classical and the quantum regimes.

Received: 28 September 2018 Accepted: 4 February 2019

Published online: 29 March 2019

\section{References}

1. Bender, C. M. \& Boettcher, S. Real spectra in non-Hermitian Hamiltonians having PT symmetry. Phys. Rev. Lett. 80, 5243-5246 (1998).

2. Bender, C. M., Boettcher, S. \& Meisinger, P. N. PT-symmetric quantum mechanics. J. Math. Phys. 40, 2201-2229 (1999).

3. Guo, A. et al. Observation of PT-symmetry breaking in complex optical potentials. Phys. Rev. Lett. 103, 93902 (2009).

4. Rüter, C. E. et al. Observation of parity-time symmetry in optics. Nat. Phys. 6, 192-195 (2010).

5. Lin, Z. et al. Unidirectional invisibility induced by PT-symmetric periodic structures. Phys. Rev. Lett. 106, 213901 (2011).

6. Feng, L. et al. Experimental demonstration of a unidirectional reflectionless parity-time metamaterial at optical frequencies. Nat. Mater. 12, 108-113 (2013).

7. Liertzer, M. et al. Pump-induced exceptional points in lasers. Phys. Rev. Lett. 108, 173901 (2012).

8. Brandstetter, M. et al. Reversing the pump dependence of a laser at an exceptional point. Nat. Commun. 5, 4034 (2014)

9. Longhi, S. Bloch oscillations in complex crystals with PT symmetry. Phys. Rev. Lett. 103, 123601 (2009).

10. $\mathrm{Xu}, \mathrm{Y}$.-L. et al. Experimental realization of Bloch oscillations in a parity-time synthetic silicon photonic lattice. Nat. Commun. 7, 11319 (2016).

11. Hodaei, H., Miri, M.-A., Heinrich, M., Christodoulides, D. N. \& Khajavikhan, M. Parity-time symmetric microring lasers. Science 346, 975-978 (2014).

12. Feng, L., Wong, Z. J., Ma, R.-M., Wang, Y. \& Zhang, X. Single-mode laser by parity-time symmetry breaking. Science 346, 972-975 (2014).

13. Miao, P. et al. Orbital angular momentum microlaser. Science 353, 464-467 (2016).

14. Gao, Z., Fryslie, S. T. M., Thompson, B. J., Carney, P. S. \& Choquette, K. D. Parity-time symmetry in coherently coupled vertical cavity laser arrays. Optica 4, 323 (2017).

15. $\mathrm{Gu}, \mathrm{Z}$. et al. Experimental demonstration of PT-symmetric stripe lasers: experimental demonstration of PT-symmetric stripe lasers. Laser Photonics Rev. 10, 588-594 (2016).

16. Bender, C. M. Making sense of non-Hermitian Hamiltonians. Rep. Prog. Phys. 70, 947-1018 (2007).

17. El-Ganainy, R. et al. Non-Hermitian physics and PTsymmetry. Nat. Phys. 14, 11-19 (2018).
18. Feng, L., El-Ganainy, R. \& Ge, L. Non-Hermitian photonics based on paritytime symmetry. Nat. Photonics 11, 752-762 (2017).

19. Konotop, V. V., Yang, J. \& Zezyulin, D. A. Nonlinear waves in PT-symmetric systems. Rev. Mod. Phys. 88, 035002 (2016).

20. Suchkov, S. V. et al. Nonlinear switching and solitons in PT-symmetric photonic systems: nonlinear PT-symmetric photonic systems. Laser Photonics Rev. 10, 177-213 (2016)

21. Chang, L. et al. Parity-time symmetry and variable optical isolation in active-passive-coupled microresonators. Nat. Photonics 8, 524-529 (2014)

22. Regensburger, A. et al. Parity-time synthetic photonic lattices. Nature 488 , 167-171 (2012)

23. Liu, Y. et al. Observation of parity-time symmetry in microwave photonics. Light Sci. Appl. 7, 38 (2018).

24. Hodaei, H. et al. Enhanced sensitivity at higher-order exceptional points. Nature 548, 187-191 (2017)

25. Chen, W., Kaya Özdemir, Ş., Zhao, G., Wiersig, J. \& Yang, L. Exceptional points enhance sensing in an optical microcavity. Nature 548, 192-196 (2017).

26. Aleahmad, P., Khajavikhan, M., Christodoulides, D. \& LiKamWa, P. Integrated multi-port circulators for unidirectional optical information transport. Sci. Rep. 7, 2129 (2017).

27. Chen, P.-Y. et al. Generalized parity-time symmetry condition for enhanced sensor telemetry. Nat. Electron. 1, 297-304 (2018)

28. Zhao, H., Chen, Z., Zhao, R. \& Feng, L. Exceptional point engineered glass slide for microscopic thermal mapping. Nat. Commun. 9, 1764 (2018).

29. Zhong, Q., Christodoulides, D. N., Khajavikhan, M., Makris, K. G. \& El Ganainy, R. Power-law scaling of extreme dynamics near higher-order exceptional points. Phys. Rev. A 97, 020105 (2018).

30. Xu, H., Mason, D., Jiang, L. \& Harris, J. G. E. Topological energy transfer in an optomechanical system with exceptional points. Nature 537, 80-83 (2016)

31. Doppler, J. et al. Dynamically encircling an exceptional point for asymmetric mode switching. Nature 537, 76-79 (2016).

32. Yoon, J. W. et al. Time-asymmetric loop around an exceptional point over the full optical communications band. Nature 562, 86-90 (2018).

33. El-Ganainy, R., Dadap, J. I. \& Osgood, R. M. Optical parametric amplification via non-Hermitian phase matching. Opt. Lett. 40, 5086-5089 (2015).

34. Zhong, Q., Ahmed, A., Dadap, J. I., JR, R. M. O. \& El-Ganainy, R. Parametric amplification in quasi-PT symmetric coupled waveguide structures. New J. Phys. 18, 125006 (2016)

35. Oulton, R. F., Sorger, V. J., Genov, D. A., Pile, D. F. P. \& Zhang, X. A hybrid plasmonic waveguide for subwavelength confinement and long-range propagation. Nat. Photonics 2, 496-500 (2008).

36. Boeck, R., Jaeger, N. A., Rouger, N. \& Chrostowski, L. Series-coupled silicon racetrack resonators and the Vernier effect: theory and measurement. Opt. Express 18, 25151 (2010).

37. Little, B. E. A variational coupled-mode theory including radiation loss for grating-assisted couplers. J. Lightware Technol. 14, 188-195 (1996).

38. Perego, A. M., Turitsyn, S. K. \& Staliunas, K. Gain through losses in nonlinear optics. Light Sci. Appl. 7, 43 (2018).

39. El-Ganainy, R., Ge, L., Khajavikhan, M. \& Christodoulides, D. N. Supersymmetric laser arrays. Phys. Rev. A 92, 33818 (2015).

40. Teimourpour, M. H., Ge, L., Christodoulides, D. N. \& El-Ganainy, R. NonHermitian engineering of single mode two dimensional laser arrays. Sci. Rep. 6, 33253 (2016)

41. St-Jean, P. et al. Lasing in topological edge states of a one-dimensional lattice. Nat. Photonics 11, 651-656 (2017).

42. Zhao, H. et al. Topological hybrid silicon microlasers. Nat. Commun. 9, 981 (2018).

43. Parto, M. et al. Edge-mode lasing in 1D topological active arrays. Phys. Rev. Lett. 120, 113901 (2018).

44. Bandres, M. A. et al. Topological insulator laser: experiments. Science 359, eaar4005 (2018)

45. Bahari, B. et al. Nonreciprocal lasing in topological cavities of arbitrary geometries. Science 358, 636-640 (2017).

46. Schindler, J., Li, A., Zheng, M. C., Ellis, F. M. \& Kottos, T. Experimental study of active LRC circuits with PT symmetries. Phys. Rev. A 84, 40101 (2011).

47. Assawaworrarit, S., Yu, X. \& Fan, S. Robust wireless power transfer using a nonlinear parity-time-symmetric circuit. Nature 546, 387-390 (2017).

48. Benisty, H. et al. Implementation of PT symmetric devices using plasmonics: principle and applications. Optics Express 19, 18004-18019 (2011).

49. Jing, H., Özdemir, Ş. K., Lü, H. \& Nori, F. High-order exceptional points in optomechanics. Sci. Rep. 7, 3386 (2017)

50. Jing, H. et al. PT-symmetric phonon laser. Phys. Rev. Lett. 113, 53604 (2014)

51. Zhang, J. et al. A phonon laser operating at an exceptional point. Nat. Photonics 12, 479-484 (2018). 


\section{Acknowledgements}

The authors thank Qi Zhong from Michigan Technological University for preparing all the figures, and acknowledge the support from National Science Foundation (ECCS 1807552, ECCS 1807485), the Army Research Office (W911NF-17-1-0481, W911NF-181-0043), Office of Naval Research (N00014-18-1-2347), Air Force Office of Scientific Research (FA9550-14-1-0037, FA9550-18-1-0235), and US-Israel Binational Science Foundation (2016381).

\section{Author contributions}

R.E-G., M.K., D.N.C., and S.K.O. contributed to the manuscript writing.

\section{Additional information}

Competing interests: The authors declare no competing interests.

Reprints and permission information is available online at http://npg.nature.com/ reprintsandpermissions/
Publisher's note: Springer Nature remains neutral with regard to jurisdictional claims in published maps and institutional affiliations.

(c) (i) Open Access This article is licensed under a Creative Commons Attribution 4.0 International License, which permits use, sharing, adaptation, distribution and reproduction in any medium or format, as long as you give appropriate credit to the original author(s) and the source, provide a link to the Creative Commons license, and indicate if changes were made. The images or other third party material in this article are included in the article's Creative Commons license, unless indicated otherwise in a credit line to the material. If material is not included in the article's Creative Commons license and your intended use is not permitted by statutory regulation or exceeds the permitted use, you will need to obtain permission directly from the copyright holder. To view a copy of this license, visit http://creativecommons.org/ licenses/by/4.0/.

(C) The Author(s) 2019 